\title{
SEARCH FOR PULSED $\gamma$-RAY EMISSION FROM GLOBULAR CLUSTER M28
}

\author{
J. H. K. Wu' ${ }^{1}$, C. Y. $\mathrm{HuI}^{2}$, E. M. H. Wu ${ }^{3}$, A. K. H. KonG ${ }^{1,4}$, \\ R. H. H. HuAnG ${ }^{1}$, P. H. T. TAM ${ }^{1}$, J. TAKATA ${ }^{3}$, AND K. S. Cheng ${ }^{3}$ \\ ${ }^{1}$ Institute of Astronomy and Department of Physics, National Tsing Hua University, Hsinchu, Taiwan; wuhkjason@gmail.com \\ ${ }^{2}$ Department of Astronomy and Space Science, Chungnam National University, Daejeon, Republic of Korea; cyhui@cnu.ac.kr \\ ${ }^{3}$ Department of Physics, University of Hong Kong, Pokfulam Road, Hong Kong \\ Received 2012 September 21; accepted 2013 February 3; published 2013 February 27
}

\begin{abstract}
Using the data from the Large Area Telescope on board the Fermi Gamma-ray Space Telescope, we have searched for $\gamma$-ray pulsations from the direction of the globular cluster M28 (NGC 6626). We report the discovery of a signal with a frequency consistent with that of the energetic millisecond pulsar (MSP) PSR B 1821-24 in M28. A weighted H-test test statistic of 28.8 is attained, which corresponds to a chance probability of $\sim 10^{-5}$ (4.3 $\sigma$ detection). With a phase-resolved analysis, the pulsed component is found to contribute $\sim 25 \%$ of the total observed $\gamma$-ray emission from the cluster. However, the unpulsed level provides a constraint for the underlying MSP population and the fundamental plane relations for the scenario of inverse Compton scattering. Follow-up timing observations in radio/X-ray are encouraged to further investigate this periodic signal candidate.
\end{abstract}

Key words: gamma rays: stars - globular clusters: individual (M28, NGC 6626) - pulsars: individual (PSR B1821-24, PSR J1824-2452A)

Online-only material: color figure

\section{INTRODUCTION}

The first millisecond pulsar (MSP), which is a rejuvenated old neutron star through accreting matter from its companion, was discovered 30 years ago (Backer et al. 1982). It has long been suggested that they are descendants of low-mass X-ray binaries (LMXBs; Alpar et al. 1982). In comparison with the Galactic field, the formation rate per unit mass of LMXBs in globular clusters (GCs) is orders of magnitude higher because of frequent stellar encounters (Katz 1975; Clark 1975; Pooley et al. 2003; Hui et al. 2010). Therefore, it is not surprising that GCs should host a large population of MSPs. Since the first cluster MSP, PSR B1821-24, was discovered in M28 (Lyne et al. 1987), dedicated radio pulsar surveys toward different clusters have resulted in the currently known population of 144 MSPs in $28 \mathrm{GCs}^{5}$

Since the launch of the Fermi Gamma-ray Space Telescope, a new population of $\gamma$-ray emitting GCs have been detected (Abdo et al. 2010a; Tam et al. 2011). As MSPs are the only known steady $\gamma$-ray sources in GCs, they are suggested to be the contributors for the observed emission. The $\gamma$-rays from a $\mathrm{GC}$ are interpreted as the collective contribution from the entire pulsar population that resides in it (Abdo et al. 2010a; Cheng et al. 2010; Hui et al. 2011). For most of the cluster MSPs, detecting the $\gamma$-ray pulsations is very challenging as the $\gamma$-ray flux of individual pulsar is weak. To exacerbate the situation, the spatial resolution of the Fermi Large Area Telescope (LAT) does not allow individual MSPs in a GC to be resolved. This results in a high background that makes the pulsation search for any individual MSP very difficult. However, there are two notable exceptions, namely, the GCs NGC 6624 and M28. Both of these GCs contain a very energetic and young MSP and thus they can possibly stand out from the background.

For PSR J1823-3021A in NGC $6624(P=5.44 \mathrm{~ms})$, its large spin-down rate, $\dot{P}=3.38 \times 10^{-18} \mathrm{~s} \mathrm{~s}^{-1}$, implies that it

\footnotetext{
4 Golden Jade Fellow of Kenda Foundation, Taiwan

5 See http://www.naic.edu/ p preire/GCpsr.html for updated information.
}

is the youngest MSP ( $\tau \sim 25 \mathrm{Myr}$ ) ever detected (Freire et al. 2011). Its spin-down luminosity is $\dot{E}=8.3 \times 10^{35} \mathrm{erg} \mathrm{s}^{-1}$, which is $\sim 1-2$ orders of magnitude greater than the typical MSPs in GCs (Bogdanov et al. 2006). Due to the accurate timing model provided by the dedicated radio observations, its $\gamma$-ray pulsations have been revealed by Fermi (Freire et al. 2011). Through a phase-resolved analysis, it has been shown that this single pulsar dominates all the observed $\gamma$-rays from NGC 6624 (Freire et al. 2011). The $\gamma$-ray conversion efficiency of PSR J1823-3021A, $L_{\gamma} / \dot{E} \sim 0.1$, is found to be comparable with other $\gamma$-ray detected MSPs (Abdo et al. 2010b), where $L_{\gamma}$ is the $\gamma$-ray luminosity.

PSR B1821-24 in M28 (hereafter M28A) is very similar to PSR J1823-3021A in many aspects. Its period $(P=3.05 \mathrm{~ms})$ and spin-down rate $\left(\dot{P}=1.61 \times 10^{-18} \mathrm{~s} \mathrm{~s}^{-1}\right)$ imply its age and spin-down power to be $\tau \sim 30 \mathrm{Myr}$ and $\dot{E}=2.2 \times 10^{36} \mathrm{erg} \mathrm{s}^{-1}$, respectively, which makes it the most energetic MSP that has been found so far (Bogdanov et al. 2011). Together with its non-thermal X-ray spectrum, its sharp and narrow X-ray pulse profile strongly indicates that most of the observed X-rays from M28A have originated from the magnetosphere. $\gamma$-ray emission from M28 has been detected by Fermi LAT (Abdo et al. 2010a). As its $L_{\gamma}$ is only a fraction of the spin-down power of M28A, it is possible that this pulsar could significantly contribute to the observed $\gamma$-rays. Together with its relatively short distance, $5.5 \mathrm{kpc}$ (cf. Harris 1996, 2010 version), the cluster M28 is a promising target for searching $\gamma$-ray pulsation. In this Letter, we report our recent search for the possible pulsation from this GC by using Fermi LAT data.

\section{DATA ANALYSIS AND RESULTS}

In this work, we used the Fermi LAT data between 2008 August 4 and 2012 January 31. For the data analysis, the Fermi Science Tools v9r23p1 package, available from the Fermi Science Support Center, ${ }^{6}$ was used. We used Pass 7 data and

\footnotetext{
6 http://fermi.gsfc.nasa.gov/ssc/data/analysis/software/
} 
Table 1

Ephemeris of PSR J1824-2452A Adopted from Ray et al. (2008)

\begin{tabular}{lc}
\hline \hline Parameter & \\
\hline Pulsar name & $\mathrm{J} 1824-2452 \mathrm{~A}$ \\
Right ascension, $\alpha$ & $18: 24: 32.00790550$ \\
Declination, $\delta$ & $-24: 52: 10.8076448$ \\
Pulse frequency, $v\left(\mathrm{~s}^{-1}\right)$ & 327.4056060517495439 \\
First derivative of pulse frequency, $\dot{v}\left(\mathrm{~s}^{-2}\right)$ & $-1.735361869603 \times 10^{-13}$ \\
Epoch of frequency determination (MJD) & 53800 \\
Epoch of position determination (MJD) & 53800 \\
Solar system ephemeris model & $\mathrm{DE} 405$ \\
Time system & $\mathrm{TDB}$ \\
\hline
\end{tabular}

selected events in the "Source" class (i.e., event class 2) only. In addition, we excluded the events with zenith angles larger than $100^{\circ}$ to greatly reduce the contamination by Earth albedo $\gamma$-rays. The instrumental response functions (IRFs) "P7SOURCE_V6" were adopted throughout the study. Events were selected within a circular region-of-interest (ROI) with a diameter of $10^{\circ}$ centered at the optical center of M28. Photon energies are restricted in the range of $200 \mathrm{MeV}-300 \mathrm{GeV}$. This set of cuts is adopted throughout this work.

To investigate the spectral characteristic of M28 with the updated IRFs and background model, we performed an unbinned likelihood analysis with the aid of gtlike by assuming a point source with power law with exponential cutoff (PLE) of the form $d N / d E \propto E^{-\Gamma} \exp \left(-E / E_{\text {cutoff }}\right)$ at the nominal position of M28, where $\Gamma$ and $E_{\text {cutoff }}$ are the photon index and the cutoff energy, respectively. To model the background, we included the Galactic diffuse model (gal_2yearp7v6_v0.fits), the isotropic background (iso_p7v6source.txt), as well as all point sources reported in the 2FGL catalog within $10^{\circ}$ from the center of the ROI. All of these 2FGL sources were assumed to be point sources which have specific spectrum suggested by the 2FGL catalog (Nolan et al. 2012). While the spectral parameters of the 2FGL sources located within the ROI were set free, we kept the parameters for those lying outside our adopted ROI fixed at the values given in 2FGL (Nolan et al. 2012). We allowed the normalizations of the diffuse background components to be free. The best-fit PLE model is characterized by $\Gamma=0.96 \pm 0.22$ and $E_{\text {cutoff }}=1.41 \pm 0.3 \mathrm{GeV}$ with a test-statistic (TS) value of 825 which is highly significant. We have tested the robustness of the spectral results by repeating the analysis with different sizes of ROI. The fitted parameters from the independent analysis are consistent within $1 \sigma$ uncertainties. In this model the photon flux between $200 \mathrm{MeV}$ and $300 \mathrm{GeV}$ was found to be $(2.39 \pm 0.22) \times 10^{-8} \mathrm{~cm}^{-2} \mathrm{~s}^{-1}$. The corresponding integrated energy flux is $f_{\gamma}=(3.17 \pm 0.29) \times 10^{-11} \mathrm{erg} \mathrm{cm}^{-2} \mathrm{~s}^{-1}$. The spectral results are consistent with those reported by Abdo et al. (2010a) within $1 \sigma$ uncertainties.

Assuming that M28A is major contributor for the $\gamma$-rays from M28, we searched for the possible $\gamma$-ray pulsation from this GC. We began by adopting the timing ephemeris determined for M28A from a long-term observation with the Rossi X-ray Timing Explorer (Ray et al. 2008), which are tabulated in Table 1. For barycentric correction, we used the updated planetary ephemeris JPL DE405 throughout this analysis. Following the method proposed by Kerr (2011), we used the best-fit model which resulted from the phase-averaged likelihood analysis as described above for assigning weight to each $\gamma$-ray photon by computing the probability that it originates from M28. This enables us to alleviate the problem of source confusion with a
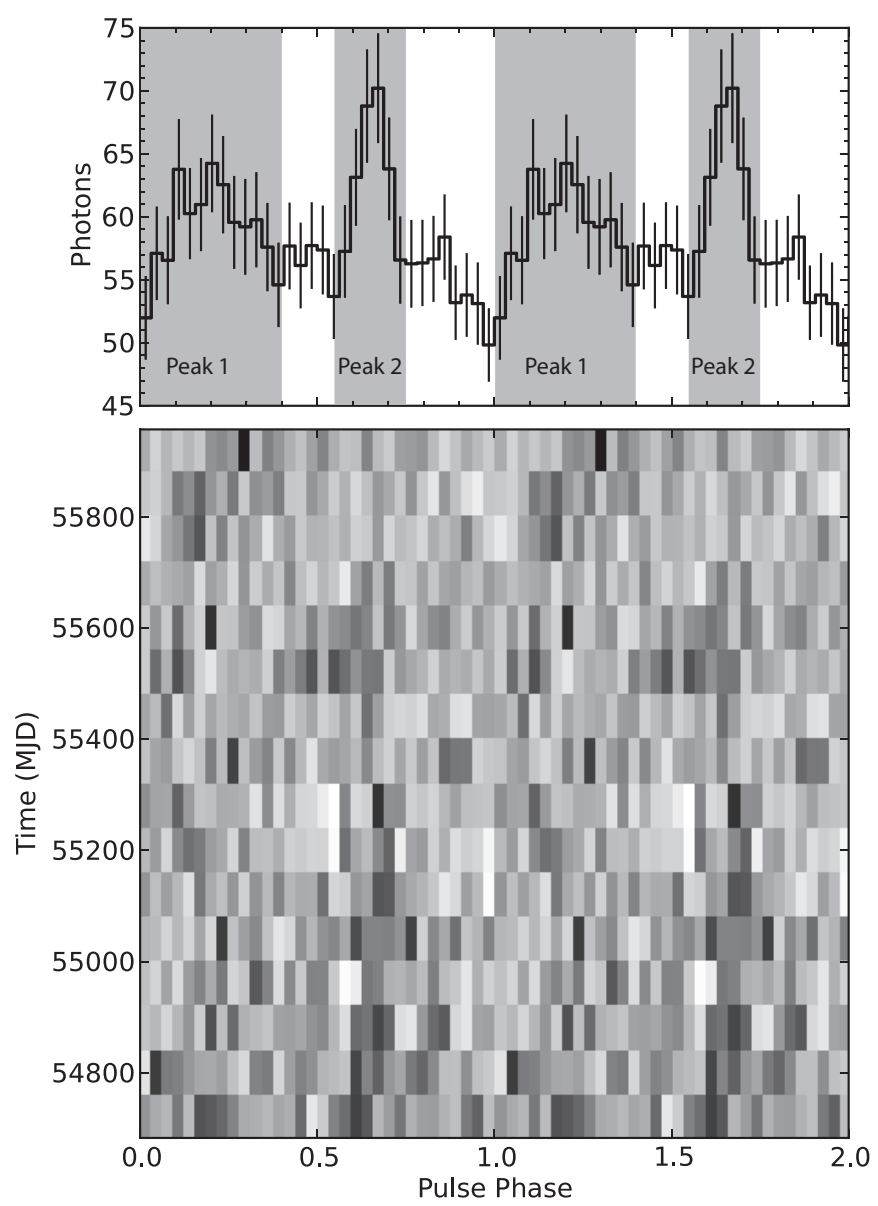

Figure 1. Fermi LAT $\gamma$-ray weighted light curve (upper panel) and the phaseogram (lower panel) of M28A. A weight was assigned to each photon with the probability that it comes from M28 by using the task gtsrcprob in the Fermi Science Tool. Two periods of rotation with a resolution of 40 phase bins per period are shown for clarity. The error bars of the light curve represent $1 \sigma$ Poisson uncertainties. The shaded regions define the on-pulse intervals for Peak 1 and Peak 2.

more efficient background rejection. We then assign a pulsar spin phase to every $\gamma$-ray photon with energies $>0.2 \mathrm{GeV}$ and within $5^{\circ}$ from M28A's direction (see Table 1). A promising signal with a weighted H-test TS of 28.8 has been found by directly folding the data with this ephemeris (de Jager $\&$ Büsching 2010). The folded $\gamma$-ray pulse profile and the phaseogram (i.e., pulse phase as a function of time) with the weighted photons are shown in the upper panel and the lower panel of Figure 1, respectively.

According to Figure 1, it appears to have two peaks, with one broader than the other. We then define the phase intervals for peak 1 and peak 2 to be $0-0.4$ and $0.55-0.75$, respectively. The rest is defined as the off-pulse component. With this definition, we show the Fermi LAT count maps of the sky region around M28 at different phases in Figure 2. During the on-pulse intervals, a point-like $\gamma$-ray source can be clearly seen at the pulsar position, which is illustrated by the yellow cross. On the other hand, a faint diffuse excess is found in the off-pulse phase (i.e., $0.40<\phi<0.55$ and $0.75<\phi<1.0$ ). However, the limited photon statistic does not allow us to constrain the extent of this putative feature.

The on-and-off nature of the $\gamma$-ray emission from M28 provides strong support for the presence of a periodic signal and leads us to a more detailed investigation. To investigate the possible spectral variations among peak 1 , peak 2 , and the unpulsed 

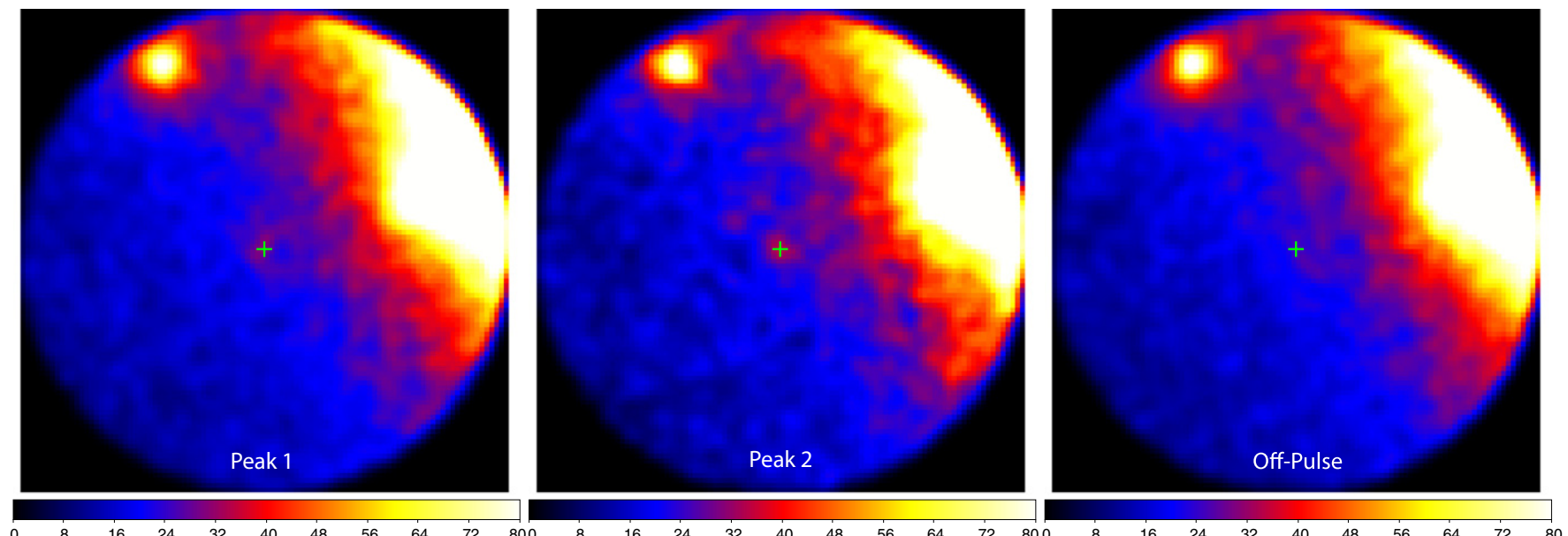

Figure 2. Fermi LAT phase-resolved $\gamma$-ray count maps for events $>0.2 \mathrm{GeV}$ within $5^{\circ}$ of the timing position of M28A (illustrated by the yellow cross). Top is north and left is east. The scale bar below shows the color scale of counts pixel ${ }^{-1}$ divided by the relevant phase interval. Left panel: Peak 1 region (i.e., $0.0<\phi<0.4$ ). Middle panel: Peak 2 region (i.e., $0.55<\phi<0.75$ ). Right panel: off-pulse region (i.e., $0.4<\phi<0.55$ and $0.75<\phi<1$ ). The point source in the northeast of these maps is 2FGL J1833.6-2104 and the bright extended emission in the northwest is due to the diffuse $\gamma$-ray emission from the Galactic plane.

(A color version of this figure is available in the online journal.)

Table 2

Phase-resolved Spectroscopy of M28A

\begin{tabular}{lccc}
\hline \hline & Peak 1 & Peak 2 & Off-pulse Component \\
\hline$\Gamma$ & $2.20 \pm 0.06$ & PL Fit & \\
$f_{\text {ph }}{ }^{\mathrm{a}}$ & $(1.45 \pm 0.14) \times 10^{-8}$ & $(9.77 \pm 1.20) \times 10^{-9}$ & $(1.02 \pm 0.13) \times 10^{-8}$ \\
$\mathrm{TS}^{3}$ & 330 & 303 & 192 \\
\hline & & PLE Fit & \\
\hline$\Gamma$ & $0.68 \pm 0.32$ & $0.96($ fixed $)$ & $1.26 \pm 0.27$ \\
$E_{\text {cutoff }}{ }^{\mathrm{b}}$ & $1.16 \pm 0.27$ & $1.21 \pm 0.12$ & $2.05 \pm 0.71$ \\
$f_{\text {ph }}{ }^{\mathrm{a}}$ & $(1.01 \pm 0.14) \times 10^{-8}$ & $(8.29 \pm 0.79) \times 10^{-9}$ & $(8.42 \pm 1.24) \times 10^{-9}$ \\
TS & 403 & 368 & 219 \\
\hline
\end{tabular}

Notes.

a Photon flux in units of photons $\mathrm{cm}^{-2} \mathrm{~s}^{-1}$ measured in the range of $0.2-300 \mathrm{GeV}$.

${ }^{b}$ Cut-off energy in units of GeV.

component, we performed a phase-resolved likelihood analysis. We fitted their spectra with both a simple power law (PL) of the form $d N / d E \propto E^{-\Gamma}$ and PLE. The results are summarized in Table 2. According to the PL fits, there is no obvious change of the spectral steepness. We note that the likelihood analysis that incorporates the PLE model results in a higher TS for all three components. For peak 2, we found that the spectral parameters for the PLE fit cannot be properly constrained. Therefore, we fixed the photon index at the value inferred in the phaseaveraged analysis (i.e., $\Gamma=0.96$ ). In the cases of the PL fits, within the tolerance of the statistical uncertainties, we do not find any conclusive evidence for the spectral variation across the phase. Assuming the off-pulse component has a constant contribution across the whole phase, $\sim 75 \%$ of the total observed flux originated from this component.

\section{SUMMARY AND DISCUSSION}

In this Letter, we report our detection of $\gamma$-ray pulsation from the direction of the GC M28. We have found a periodic signal which presumably originated from its energetic MSP M28A. Based on our phase-resolved analysis, the pulsed component contributes $\sim 25 \%$ of the total observed $\gamma$-rays. At a distance of $d=5.5 \mathrm{kpc}$, this implies an on-pulse luminosity of $L_{\gamma}=4 \pi d^{2} f_{\Omega} f_{\gamma} \sim 3 \times 10^{34} f_{\Omega} \mathrm{erg} \mathrm{s}^{-1}$, where $f_{\Omega}$ is the fraction of the sky covered by the $\gamma$-ray beam. Assuming that the pulsed emission originated from M28A, this suggests a $\gamma$-ray conversion efficiency of $L_{\gamma} / \dot{E} \sim 0.01 f_{\Omega}$. For some of the MSPs in the Galactic field, such as PSRs J2124-3358 and J0437-4715, we find $L_{\gamma} / \dot{E}$ at this level (Abdo et al. 2010b). However, this is lower than $L_{\gamma} / \dot{E} \sim 0.08$ as derived from the nearby MSPs (Abdo et al. 2009). If one adopts this as the intrinsic $\gamma$-ray conversion efficiency of M28A, this might suggest that the observed period derivative is largely dominated by the acceleration of a pulsar along the line of sight due to the gravitational field of the cluster.

On the other hand, the off-pulse luminosity is found at the level of $L_{\gamma} \sim 8 \times 10^{34} \mathrm{erg} \mathrm{s}^{-1}$. This estimate is useful for constraining the collective properties of the rest of the MSP population in M28. There are two main theories to explain the unpulsed $\gamma$-ray emission from GCs. One theory proposes that the $\gamma$-ray emission from a GC originated from the collection of the magnetospheric radiation from the entire MSP population that resides in it (Abdo et al. 2010a; Venter \& de Jager 2008; Venter et al. 2009). Assuming an average spin-down power of $\langle\dot{E}\rangle \sim 2 \times 10^{34} \mathrm{erg} \mathrm{s}^{-1}$ and a characteristic conversion efficiency of $\sim 0.08$, the off-pulse luminosity enables us to estimate the number of the rest MSP population to be $N_{\mathrm{MSP}} \sim 50$. This suggests that about one-fifth of the underlying population has already been uncovered.

Besides the aforementioned standard scenario, inverse Compton scattering (ICS) between the relativistic pulsar wind particles and the ambient soft photons has also been proposed as another possible explanation for the origin of the $\gamma$-ray from GCs (Bednarek \& Sitarek 2007; Cheng et al. 2010; Hui et al. 2011). Cheng et al. (2010) found that the observed $\gamma$-ray spectra of GCs can generally be well modeled by ICS between the $e^{-} / e^{+}$in the pulsar wind of the whole MSP population in a GC and the Galactic background IR photons or starlight. The two-dimensional regression analysis further suggests $L_{\gamma}$, energy density of the background optical/IR photon field, and the stellar encounter rate/metallicity span a set of fundamental planes (Hui et al. 2011). The unpulsed level inferred for M28 can be used to discriminate which relation(s) can better predict the collective 
contribution. Using the best-fit parameters for these fundamental plane relations (Equations (1)-(4) and Table 3 in Hui et al. 2011) and the updated GC parameters (Harris 1996, 2010 version), the relations involve metallicity and optical/IR energy densities result in an estimate in a range of $\sim(8-9) \times 10^{34} \mathrm{erg} \mathrm{s}^{-1}$, which is consistent with the observed off-pulse luminosity. On the other hand, the best-fit relations that involve the encounter rate result in an estimate of $\sim 2 \times 10^{35} \mathrm{erg} \mathrm{s}^{-1}$ which apparently overshoots the observed value.

To further investigate this putative periodic signal, multiwavelength observations are certainly required. In particular, the phase-aligned $\mathrm{X}$-ray $/ \gamma$-ray pulse profile will provide an important constraint for the high-energy emission model. However, no existing X-ray timing data are available for M28A in the Fermi era. As the timing noise of M28A is quite strong in comparison with other MSPs and it possibly exhibited glitches, the phase alignment of multi-wavelength light curves subjects to a lot of uncertainties. Therefore, follow-up timing observations in other wavelengths are encouraged for further investigations.

The authors thank Paul Ray and the anonymous referee for providing a code for computing phaseogram and useful comments for improving the quality of this manuscript. This project is supported by the National Science Council of the Republic of China (Taiwan) through grants NSC100-2628-M-007-002MY3 and NSC100-2923-M-007-001-MY3. C.Y.H. is supported by the National Research Foundation of Korea through grant 2011-0023383. A.K.H.K. gratefully acknowledges support from a Kenda Foundation Golden Jade Fellowship. J.T. and K.S.C. are supported by a GRF grant of the HK Government under HKU700911P

\section{REFERENCES}

Abdo, A. A., Ackermann, M., Ajello, M., et al. 2009, Sci, 325, 848

Abdo, A. A., Ackermann, M., Ajello, M., et al. 2010a, A\&A, 524, 75

Abdo, A. A., Ackermann, M., Ajello, M., et al. 2010b, ApJS, 187, 460

Alpar, M. A., Cheng, A. F., Ruderman, M. A., \& Shaham, J. 1982, Natur, 300,728

Backer, D. C., Kulkarni, S. R., Heiles, C., Davis, M. M., \& Goss, W. M. 1982, Natur, 300, 615

Bednarek, W., \& Sitarek, J. 2007, MNRAS, 377, 920

Bogdanov, S., Grindlay, J. E., Heinke, C. O., et al. 2006, ApJ, 646, 1104

Bogdanov, S., van den Berg, M., Servillat, M., et al. 2011, ApJ, 730, 81

Cheng, K. S., Chernyshov, D. O., Dogiel, V. A., Hui, C. Y., \& Kong, A. K. H 2010, ApJ, 723, 1219

Clark, G. W. 1975, ApJL, 199, L143

de Jager, O. C., \& Büsching, I. 2010, A\&A, 517, L9

Freire, P. C. C., Abdo, A. A., Ajello, M., et al. 2011, Sci, 334, 1107

Harris, W. E. 1996, AJ, 112, 1487 (2010 version)

Hui, C. Y., Cheng, K. S., \& Taam, R. E. 2010, ApJ, 714, 1149

Hui, C. Y., Cheng, K. S., Wang, Y., et al. 2011, ApJ, 726, 100

Katz, J. I. 1975, Natur, 253, 698

Kerr, M. 2011, ApJ, 732, 38

Lyne, A. G., Brinklow, A., Middleditch, J., Kulkarni, S. R., \& Backer, D. C. 1987, Natur, 328, 399

Nolan, P. L., Abdo, A. A., Ackermann, M., et al. 2012, ApJS, 199, 31

Pooley, D., Lewin, W. H. G., Anderson, S. F., et al. 2003, ApJL, 591, L131

Ray, P. S., Wolff, M. T., Demorest, P., et al. 2008, in AIP Conf. Proc. 983, 40 Years of Pulsars: Millisecond Pulsars, Magnetars and More, ed. C. Bassa et al. (Melville, NY: AIP), 157

Tam, P. H. T., Kong, A. K. H., Hui, C. Y., et al. 2011, ApJ, 729, 90

Venter, C., \& de Jager, O. C. 2008, ApJL, 680, L125

Venter, C., de Jager, O. C., \& Clapson, A.-C. 2009, ApJL, 696, L52 